\title{
Histological and morphological studies of cardiac cells in response to aerobic exercise and rosemary extract in rat model of aging
}

\author{
Shima Ababzadeh ${ }^{1}$ Amir Iravani ${ }^{2}$ Faranak Fallahian ${ }^{3}$ Seyed Mahdi Aghamiri ${ }^{2}$ \\ ${ }^{1}$ Department of Anatomy, Faculty of Medicine, Qom University of \\ Medical Sciences, Qom, Iran \\ 2 Student Research Committee, Faculty of Medicine, Qom University \\ of Medical Sciences, Qom, Iran \\ Address for correspondence Seyed Mahdi Aghamiri, Student \\ Research Committee, Faculty of Medicine, Qom University of Medical \\ Science and Health Services, number 9, attaran st, Qom, Iran \\ (e-mail: smaghamiri113@gmail.com).
}

${ }^{3}$ Department of Biochemistry, Faculty of Medicine, Qom University of Medical Sciences, Qom, Iran

J Morphol Sci 2018;35:266-271.

\begin{abstract}
Objective The average age of the world population is growing increasingly. Since aging is the biggest risk factor for cardiac diseases, the incidence of these types of disease will increase significantly in the near future. However, their occurrence may be prevented by some factors, such as the practice of aerobic exercise and the use of herbal medicine.

Materials and methods Thirty male Wistar rats, at 18 months old each, were divided into 5 groups $(n=6)$ : control (NO), gavage (GO), exercised (EO), extract-fed (EXO), and exercised and extract-fed (EEO). The animals of the $\mathrm{GO}$ group received distilled water and were put on a silent electric running machine. The animals of the EO and EEO groups were assigned to a treadmill training protocol at low and moderate intensities for 1 hour/day, 5 days/week for 12 weeks. The animals of the EXO and EEO groups were treated with rosemary (Rosmarinus officinalis) extract for 12 weeks. At the end of the experiment, the hearts of the rats were collected and submitted to hematoxylin and eosin and Mallory trichrome staining. Images of these stained samples were captured, and a morphometric analysis of the cardiac tissue was performed.

Results The rats in the EO, EXO and EEO groups showed a reduction in the intercellular space when compared with those of the NO and GO groups. The rats in the EO and EEO groups showed an increase in the clarification of the intercalated discs and in the width of the cardiomyocytes, when compared with those in the other groups. The nuclei of the heart cells of the animals in the EXO group were seen as more euchromatic than others.
Keywords
Conclusion Aerobic exercise causes changes in the cardiac muscles of rats and
- aging
- aerobic exercise
- rosemary extract
- rat confirms the capacity of the fibers to adapt to the requirements of the proposed aerobic exercise. The antioxidants found in rosemary extract improve cell activity to achieve a better performance. However, applying these two factors simultaneously indicates the reduction of their positive influences.

received

July 24, 2018

accepted

October 5, 2018
DOI https://doi.org/

10.1055/s-0038-1676778.

ISSN 2177-0298.
Copyright (e 2018 by Thieme Revinter

Publicações Ltda, Rio de Janeiro, Brazil
License terms

(c) $(1) \$$ 


\section{Introduction}

The aging of the population and its consequences is one of the greatest social problems in most of the countries in the world. The number of people $>65$ years old was estimated at 524 million in 2010, and it will reach 1.5 billion until 2050 . $^{1}$

Old age is said to be a series of time-dependent anatomic changes. ${ }^{2}$ During old age, all of the organs, including the heart, undergo changes at the morphological and physiological levels. The physiological changes include: reduction in the maximum efficiency of the heart, reduction in the diastolic efficiency, higher incidence of both brady- and tachyarrhythmias, reduction in the maximum intake of calcium and oxygen, and reduction in heart capacity during exercise due to fibrosing of the atrium, which is one of the main reasons of heart diseases in old age. ${ }^{3}$ Morphological changes include: increase of the diameter of the wall of the heart, especially of the left ventricle, hypertrophy of cells, as well as of the extracellular and fibrosis matrices, reduction of the number of cells, and lipofuscin accumulation in the cells. ${ }^{3,4}$

Therefore, by presenting ways to postpone the aging process, it is possible to avoid several fatal diseases caused by aging, such as heart disability. ${ }^{5}$ Many of the drug interventions to avoid and cure cardiovascular diseases cause inhibition and are accompanied by pathological stress. Therefore, other interventions to solve this problem, including the practice of exercises and the use of medicinal plants, deserve attention. ${ }^{6}$ Cardiomyocytes are inseparable, ${ }^{7}$ but when confronting pathological and physiological stimuli, they can be revived and enabled to separate. ${ }^{8}$ One of the most important and cheapest ways to safely provide physiological stimuli to revive the heart cells is practicing aerobic exercise, which changes the metabolic conditions of heart cells in a desirable way and causes the activation of reparative mechanisms. According to previous researches, aerobic exercise has some effects, including the increase in the number of the heart muscle cells, the increase in the number of precursor cells for restorative actions, the increase in the volume of the left ventricle, the increase of the spastic power of the heart, the increase of heart efficiency, a protective effect against oxidative stress, ${ }^{9}$ a change in the nature of the extracellular matrix of the heart tissue, and reduction of fibrosis. ${ }^{10}$ It should also be noted that there are several factors regarding the positive effects of aerobic exercise, which consist of positve effects on the smooth muscles of the peripheral vessels and in the metabolism, as well as a reduction in cardiovascular risk factors, and improvements in diabetes and in blood pressure. ${ }^{9}$

Another intervention for the improvement of the health of the heart is the use of medicinal plants. Rosemary (Rosmarinus officinalis), a plant with thin leaves and purple-blue flowers, is a member of the Lamiaceae family and of the group of medicinal plants. More accurately, rosemary extract consists of volatile oil materials, ${ }^{11}$ including several antioxidants, such as rosemarinic acid, caffeic acid, chlorogenic acid, carnosic acid, rosmanol, carnosol, rosemary diphenol, rosemary quinone, as well as other natural antioxidants, such as ursolic acid, glucocolic acid, alkaloid rosmaricine, etc. Carnosic acid and carnosol are the most powerful antiox- idants of this plant. Rosemary has a potential property to cure many diseases, including inflammatory diseases and ischemia in heart diseases. It also has an antioxidant property that inhibits mutations in the DNA, and it is able to neutralize the negative effect of free radicals on different cells. $^{12}$ By reviewing others articles, and since no study reviewed all of the aging factors, along with the practice of aerobic exercise concurrently with the use of rosemary extract, we decided to put old mice in different groups and treat them separately and simultaneously with aerobic exercise and rosemary extract and review the effects of these two factors, besides aging, on their heart tissue and on the morphological properties of their heart cells.

\section{Materials and Methods}

Staining: Hematoxylin and eosin, and Mallory trichrome.

Extract: Rosemary extract including $40 \%$ of carnosic acid (Hunan Geneham Biomedical Technology Ltd., Hunan, Changsha, China).

Aerobic Exercise protocol: At first, to introduce the treadmill device to the rats, we made them exercise with a low speed (10m/minute) for 10 minutes for 7 days. Afterwards, we grouped the rats randomly. We divided the animals in exercised (EO) and exercised and extract-fed (EEO) groups and made them exercise on a 0 -degree slope for 1 hour/day for 12 weeks, 5 days per week, after that the treadmill speed was increased from $12 \mathrm{~m} /$ minute to $22 \mathrm{~m} /$ minute.

\section{Animal Work (Samples)}

In the present study, 12 -month-old male Wistar Rats $(n=30)$ weighing between 300 and $350 \mathrm{~g}$ were brought from the Center of Animal Breeding of the University of Iran. We took care of the 12-month-old rats under standard conditions of food and water and 12-hours light-dark cycles for 6 months until they reached the age of 18 months old (average weight: 350-400 g). Afterwards, the old rats were randomly divided into the following 5 groups ( $n=6$ for each group):

1. Control group (NO).

2. Gavage group (GO): the group that received the solvent of rosemary's extract (Distilled water) and was put on a turned-off treadmill for 10 minutes daily.

3. Exercised group (EO): the group that was put on a turnedon treadmill based on an exercise protocol for 3 months (12 weeks).

4. Extract-fed (EXO): the group that received rosemary extract (100 mg/kg) daily through a gavage for 3 months (12 weeks).

5. Exercised and extract-fed (EEO): the group that received rosemary extract $(100 \mathrm{mg} / \mathrm{kg}$ ) daily through a gavage for 3 months (12 weeks) and was concurrently put on a turnedon treadmill based on an exercise protocol.

\section{Tissue Preparation}

After sacrificing the rats, we separated their heart tissue and moved it to dishes containing $10 \%$ formalin to stabilize it. After stabilizing the samples and obtaining $5 \mu \mathrm{m}$ tissue sections with a Leica RM2235 microtome (Leica Biosystems, 
Wetzlar, Germany), they were stained with hematoxylin and eosin and with Mallory trichrome according the protocol presented below.

\section{Hematoxylin \& Eosin Staining}

We placed the glass slides that held the paraffin sections in staining racks. Then, we cleared the paraffin from the samples in 3 changes of xylene for 2 minutes per change. The sections were dehydrated through 3 changes of $100 \%, 95 \%$, and $70 \%$ ethanol, and were submerged in water for 2 minutes per change. The nuclei were stained in hematoxylin solution for 3 minutes. Then, they were carefully washed in tap water at room temperature for at least 5 minutes, and were differentiated in 1\% acid alcohol (1\% hydrochloric acid [HCL] in 70\% alcohol) for between 5 and 10 seconds. Afterwards, the sections were carefully washed in tap water for between 10 and 15 minutes until they were blue again. The samples were stained in working eosin Y solution for 2 minutes. Then, they were carefully washed in tap water. Dehydrated through alcohols, $95 \%$, two changes of $100 \%$ ethanol for 2 minutes per change. So The Cleared in xylene 3 changes of xylene for 2 minutes per change and Mounted in permanent mounting medium. The slides were viewed using a microscope (Leica Microsystems, Wetzlar, Germany). After staining, we chose 5 laminas from each group and captured several images from the heart muscles with different magnifications. Then, we selected 5 grade views with the ImageJ software and elected the fibers located on adjacent sides to do the morphological and morphometric review. ${ }^{13}$

\section{Mallory Trichrome Staining}

The deparaffinized sections were hydrated through graded alcohols to water. The nuclei were stained in hematoxylin solution for 3 minutes. Afterwards, they were differentiated in $1 \%$ acid alcohol ( $1 \%$ HCL in $70 \%$ alcohol) for between 5 and 10 seconds. The sections were carefully washed in tap water for between 10 and 15 minutes until they were blue again. They Stained in acid fushion solution 0.5\%, 5 minutes Rinse in distilled water. Stain with aniline blue/orange G solution, 20 minutes. Rinse in distilled water. Dehydrate through alcohols, clear, and mount. ${ }^{13}$

\section{Morphological Review (Qualitative Review)}

In the present review, changes in the morphology of the tissue, such as whole structure of heart cells in $\mathrm{x} 4$ and $\mathrm{x} 40$ magnifications, and striated lines of the heart cells qualitatively were considered at x1,000 magnification.

\section{Morphometric Review (Quantitative Review)}

In this section, we examined the heart cells with $\mathrm{x} 400$ magnification; we reviewed their situation with each other, diameter changes and changes in the degree of coloration of the cell nucleus with the ImageJ software for each animal.

\section{Results}

The microscopic views of the longitudinal sections of the heart fibers are shown in -Figs. 1 and 2.

The review of the optical microscope micrographs in a qualitative perspective evidenced the existence of empty spaces (shown by black arrows) between the cardiomyocytes in the NO and GO groups. However, in the striated lines of the heart cells sections of the EO, EEO, and EXO groups, this space is reduced and less empty space is observed. Another factor that was observed in a qualitative way was the transparency of lateral lines. These lines were more obvious in the EO and in the EEO groups.

To review the micrographs of optical microscope in a quantitative view, the review of the diameter of heart tissues and of the amount of brightness of the core of the heart tissues was used to review the achievement of more euchromatinization and heterochromatinization. The results are shown in -Figs. 3 and 4.

In previous studies, the diameters of heart fibers are averagely meaningful in the EO, and in the EEO groups.

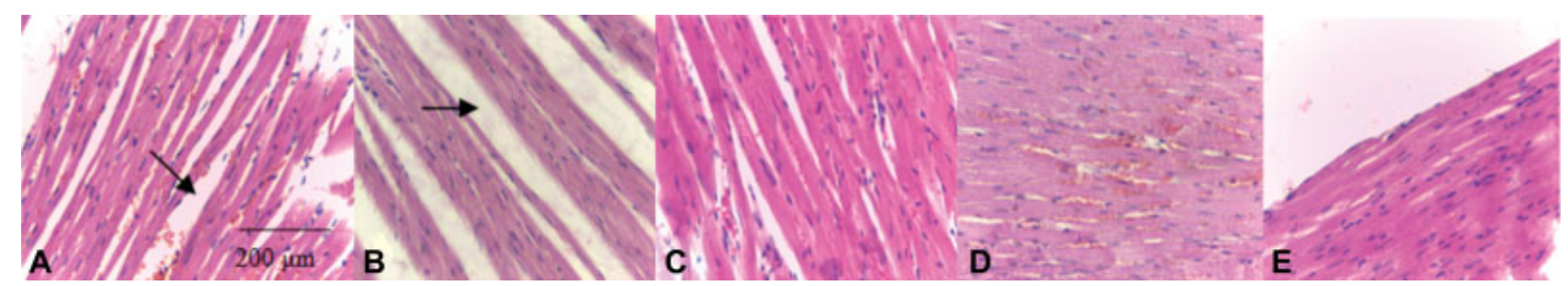

Fig. 1 Photomicrographs of heart tissue in the A, control (NO); B, gavage (GO); C, exercised (EO); D, extract-fed (EXO); and E, exercised and extract-fed (EEO) groups. Stained with hematoxylin and eosin ( $\times 400$ magnification). The black arrows show the space between the cells in the $\mathrm{NO}$ and GO groups.

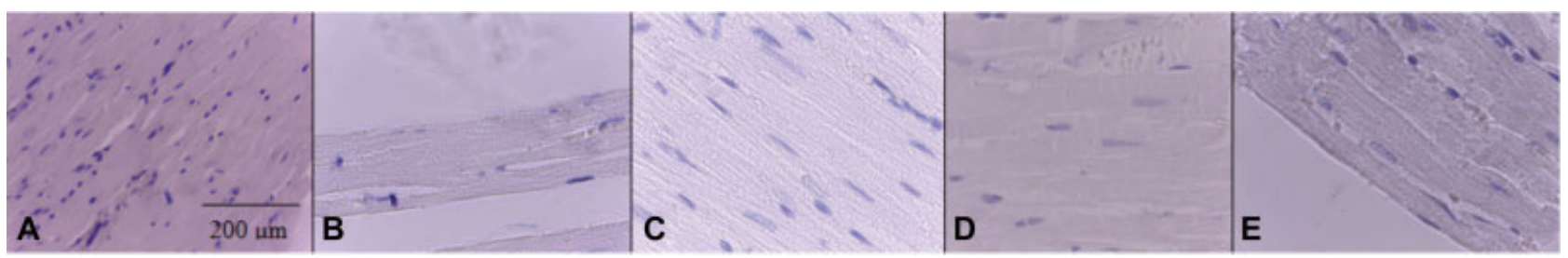

Fig. 2 Photomicrographs of heart tissue in A, control (NO); B, gavage (GO); C, exercised (EO); D, extract-fed (EXO); and E, exercised and extractfed (EEO) groups. Stained with Mallory trichrome ( $\times 400$ magnification). 


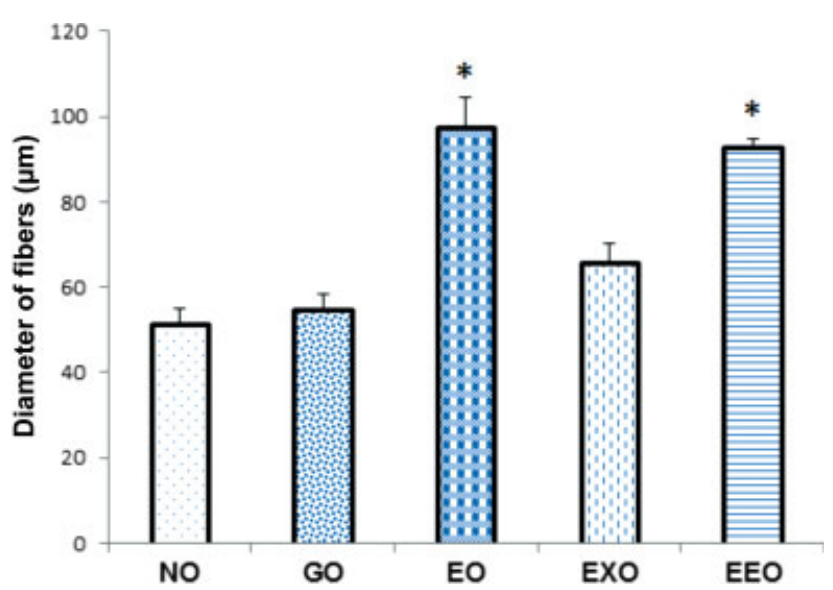

Fig. 3 Average diameter $(\mu \mathrm{m})$ of the heart fibers of the studied groups . Data are presented in mean \pm standard error of the mean (SEM). * ( $p 0.05)$ : significant difference with the control group. Control (NO), gavage (GO), exercised (EO), extract-fed (EXO), and exercised and extract-fed (EEO) groups.

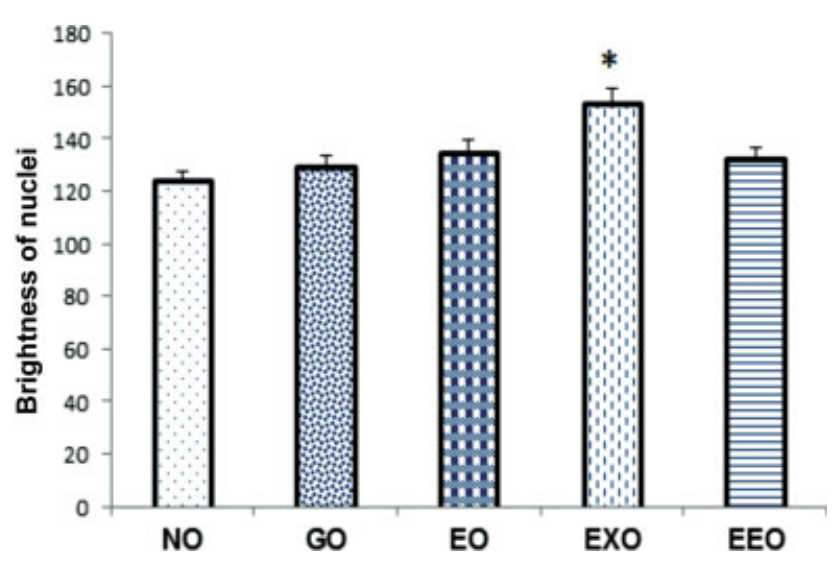

Fig. 4 Average amount of heart fibers core light of the studied groups. Data are presented in Mean \pm standard error of the mean (SEM). * ( $p$ 0.05): significant difference with the control group. Control (NO), gavage (GO), exercised (EO), extract-fed (EXO), and exercised and extract-fed (EEO) groups.

This shows the positive effect of aerobic exercise on the increase in the number of heart fibers and kind of revival of these fibers in old ages.

In the study of the euchromatinization of the nuclei of heart fibers, the EXO group was significantly more euchromatinized and brighter, which can indicate the reactivity of the cell in front of aging factor which leads it to darkness and being more heterochromatinized and of course this effect is mostok probably caused by the great antioxidant property of rosemary extract and its removal of free radicals. (-Tables $\mathbf{1}$ and $\mathbf{2}$ )

\section{Statistical Analysis of Data}

Data was analyzed by SPSS Statistics for Windows, Version 16.0 (SPSS Inc, Chicago, IL, USA) and by the analysis of variance (ANOVA) test. The differences were considered significant when $p$ 0.05. The charts were drawn by Microsoft Excel 2010 (Microsoft Corporation, Redmond, WA, USA).
Table 1 Average diameter of the heart fibers of the studied groups

\begin{tabular}{|l|l|l|}
\hline Group & Mean & SEM \\
\hline NO & 51.54 & 3.3 \\
\hline GO & 54.98 & 3.5 \\
\hline EO & 97.71 & 6.8 \\
\hline EXO & 66 & 4.4 \\
\hline EEO & 92.99 & 1.9 \\
\hline
\end{tabular}

Abbreviations: EEO, exercised and extract-fed group; EO, exercised group; EXO, extract-fed group; GO, gavage group; NO, control group; SEM, standard error of the mean.

$\left(\begin{array}{ll}p & 0.05\end{array}\right)^{* *}$ : meaningful difference with the control group.

Table 2 Average amount of heart fibers core light of the studied groups

\begin{tabular}{|l|l|l|}
\hline Groups & Mean & SEM \\
\hline NO & 124.2 & 3.7 \\
\hline GO & 129.6 & 4.13 \\
\hline EO & 135.2 & 4.07 \\
\hline EXO & 154.2 & 4.63 \\
\hline EEO & 132.6 & 3.77 \\
\hline
\end{tabular}

Abbreviations: EEO, exercised and extract-fed group; EO, exercised group; EXO, extract-fed group; GO, gavage group; NO, control group; SEM, standard error of the mean.

(p 0.05).**: meaningful difference with the control group.

\section{Discussion}

The remarkable increase in the average age of the population in this century is caused by an improvement in health worldwide, by the discovery of cures for many parasitic and infectious diseases, by improvements in diet, and by higher rates of vaccination. According to epidemiological studies, in recent years, the demographics of diseases have changed from harsh contagious infectious diseases to noncontagious degenerative and chronic diseases. Heart diseases have a great importance so that 40 percent of deaths is assigned to people over $65 .{ }^{1}$ In old age, some changes occur in the heart tissue that make the context for heart diseases and impress the efficiency of this important organ. Managing these changes and postponing them is possible due to factors such as the practice of aerobic exercise and the use of medicinal plants.

Doing aerobic exercise is one of the most effective ways to improve the efficiency of different organs of the body, especially of the heart. Therefore, in previous studies, effects such as positive physiologic anatomic changes, improvements in the efficiency of the heart and in the maximum oxygen absorption, reduction in the oxidative stress, quality control of heart proteins ${ }^{14,15}$ and, in some cases, sudden mutations, were observed. ${ }^{16}$ In most studies, the physiologic effects of aerobic exercise were evaluated. However, in the present study, the histologic effects of aerobic exercise on the heart tissues were reviewed, and it was observed that this 
factor causes a significant increase in the number of heart fibers, a non-significant euchromatinic increase of the nuclei, a reduction in the intercellular space, and an increase in the transparency of the lateral lines of the muscle fibers of the heart. All of these factors confirm the improvement of the morphological structure of the cells and of the muscle structure of the heart of the exercised groups in comparison with the unexercised groups.

Another factor that makes it possible to manage the changes of heart tissue in old age is the use of medicinal plants, such as rosemary, which was used in the present study. The leaves of $R$. officinalis possess a variety of bioactive agents, including antioxidants, antitumorals, and antiinflammatories. The main relevant constituents are composed of a vast number of polyphenolics, including carnosic acid, carnosol, rosemarinic acid, and ursolic acid, among others. Carnosic acid (a phenolic diterpene compound) and carnosol are the most potent among these antioxidant constituents (responsible for $\sim 90 \%$ of the antioxidant activity of rosemary extract).Rosemary extract has positive effects, such as the reduction of the harm caused by free radicals and inflammatory factors, ${ }^{11}$ free radicals repulse increase, ${ }^{17}$ and the reduction of the amount of nitric oxide synthase (NOS) and carboxyltransferase (CT) enzymes. ${ }^{18}$ Nevertheless, there is very little information about the effect of rosemary extract on the structure of the tissues of old hearts. In the present study, rosemary extract caused euchromatinization of the core of heart cells in old mice.

In some cases, the performance of aerobic exercise concurrently with the use of medicinal plants intensifies the effect these factors, and, in other cases, lessens the effect of each factor. Previous researches on the benefits of aerobic exercises and medicinal plants show the positive effects on the antioxidant system and the increase of catalase gene expression and of glutathione peroxidase. ${ }^{19}$ Also, in some studies, no significant beneficial effects of performing aerobic exercise concurrently with the use of medicinal plants were observed. ${ }^{20}$ In the present study, considering that no previous study had ever reviewed the simultaneous effect of aerobic exercise and rosemary extract on the tissue structure of the hearts of old mice, it was observed that, in the EEO group, the space between the tissues is reduced in a qualitative perspective, inflammatory cells are rarely seen, and the lateral lines have become clearer than those in the NO group; also, in the EEO group, the diameter of the heart fibers increased significantly in a quantity perspective, and the nuclei were euchromatinized, but not significantly.

Therefore, according to the data of the present study, the simultaneous review of intervention factors shows that these factors can reduce the effect of each other according to the charts of the diameter of the muscular fibers and of euchromatinic nuclei.

\section{Conclusion}

Aerobic exercise causes changes in the cardiac muscles of rats and confirms the capacity of the fibers to adapt to the requirements of the proposed aerobic exercise. Rosemary extract improves cell activity by means of its antioxidants to achieve a better performance. However, applying these two factors synthetically indicates the reduction of their positive influences.

\section{Conflicts of Interest}

The authors have no conflicts of interest to declare.

\section{Acknowledgment}

The present study was supported by a grant from the vice chancellor of research of the Qom University of Medical Sciences (MUQ.REC.1395.23).

\section{References}

1 Pardon MC. Stress and ageing interactions: a paradox in the context of shared etiological and physiopathological processes. Brain Res Brain Res Rev 2007;54(02):251-273

2 Hung CW, Chen YC, Hsieh WL, Chiou SH, Kao CL. Ageing and neurodegenerative diseases. Ageing Res Rev 2010;9(Suppl 1): S36-S46

3 Chiao YA, Rabinovitch PS. The aging heart. Cold Spring Harb Perspect Med 2015;5(09):a025148

4 Juhaszova M, Rabuel C, Zorov DB, Lakatta EG, Sollott SJ. Protection in the aged heart: preventing the heart-break of old age? Cardiovasc Res 2005;66(02):233-244

5 Harman D. Free radical theory of aging. Mutat Res 1992;275(36):257-266

6 Mann N, Rosenzweig A. Can exercise teach us how to treat heart disease? Circulation 2012;126(22):2625-2635

7 Godfrey R, Theologou T, Dellegrottaglie S, et al. The effect of highintensity aerobic interval training on postinfarction left ventricular remodelling. BMJ Case Rep 2013;2013:bcr2012007668

8 Boström P, Mann N, Wu J, et al. C/EBP $\beta$ controls exercise-induced cardiac growth and protects against pathological cardiac remodeling. Cell 2010;143(07):1072-1083

9 da Silva MF, Natali AJ, da Silva E, et al. Attenuation of Ca2+ homeostasis, oxidative stress, and mitochondrial dysfunctions in diabetic rat heart: insulin therapy or aerobic exercise? J Appl Physiol (1985) 2015;119(02):148-156

10 Kwak HB. Aging, exercise, and extracellular matrix in the heart. J Exerc Rehabil 2013;9(03):338-347

11 al-Sereiti MR, Abu-Amer KM, Sen P. Pharmacology of rosemary (Rosmarinus officinalis Linn.) and its therapeutic potentials. Indian J Exp Biol 1999;37(02):124-130

12 Alnahdi HS. Effect of Rosmarinus officinalis extract on some cardiac enzymes of streptozotocin-induced diabetic rats. J Health Sci (Sarajevo) 2012;2(04):33-37

13 Bancroft JD, Gamble M. Theory and practice of histological techniques. Elsevier Health Sciences; 2008

14 Dorr A, Thomason LA, Koletar MM, et al. Effects of voluntary exercise on structure and function of cortical microvasculature. J Cereb Blood Flow Metab 2017;37(03):1046-1059

15 Campos JC, Queliconi BB, Dourado PM, et al. Exercise training restores cardiac protein quality control in heart failure. PLoS One 2012;7(12):e52764

16 Waring CD, Vicinanza C, Papalamprou A, et al. The adult heart responds to increased workload with physiologic hypertrophy, cardiac stem cell activation, and new myocyte formation. Eur Heart J 2014;35(39):2722-2731

17 Botsoglou NA, et al. Effect of long-term dietary administration of oregano and rosemary on the antioxidant status of rat serum, liver, kidney and heart after carbon tetrachloride-induced oxidative stress. J Sci Food Agric 2009;89(08):1397-1406 
18 Posadas SJ, Caz V, Largo C, et al. Protective effect of supercritical fluid rosemary extract, Rosmarinus officinalis, on antioxidants of major organs of aged rats. Exp Gerontol 2009;44(6-7): 383-389

19 Katoli M, Daloii AA. The effect of Boldenone and Aerobic Training with Jujube Extract and Gallic Acid on Glutathione Peroxidase and
Catalase in Heart Tissue of Male Wistar Rats. Traditional and Integrative Medicine 2017;2(01):15-23

20 Razavimajd Z, Homaee HM, Azarbayjani MA, Farzanegi P. The Effect of Regular Aerobic Exercise with Garlic Extract on Heart Apoptosis Regulatory Factors in Chronic Kidney Disease. Iranian J Diabetes Obesity 2017;9(1-2):62-68 